\title{
Quelques travaux de correction sur le cours de l'Isère
}

PAR

M. Blanic

Ingenieur en Chef des Ponts et Chaussées
ET

\author{
G. Verdet
}

Ingénieur SOGREAH

\section{1 - Introduction}

Dans les vallées alluviales, le cours d'eau a toujours été un facteur primordial des conditions de vie des populations. $\mathrm{Si}$ pendant longtemps l'Egypte a vécu au rythme du Nil dont la crue fertilisait chaque année la vallée, dans les vallées alpestres, par contre, rivières et torrents ont toujours eu des effets dévastateurs contre lesquels les riverains essayaient de se protéger. Dans bien des cas les endiguements, travaux de protection ou de correction ont été entrepris pour combattre un environnement précaire dû aux divagations des lits des cours d'eau. C'est ainsi que l'« environnement "n'a souvent pas seulement à être sauvegardé, mais aussi à être aménagé, amélioré.

La vallée de l'Isère, dans le «sillon Alpin » et jusqu'à l'aval de la cluse de Voreppe, est un bon exemple de l'effort que l'homme a déployé pour aménager le fond des vallées au détriment du domaine dévolu jadis à la rivière. Depuis plus d'un siècle et demi, elle a en effet, été l'objet de travaux considérables.

$\mathrm{Si}$ les premiers endiguements, réalisés empiriquement et avec des moyens limités, ont pu présenter des effets secondaires néfastes, les méthodes d'études qui se sont développées depuis lors, ainsi que la puissance des engins mécaniques modernes, permettent d'envisager des solutions beaucoup plus sûres et radicales que par le passé.

Après une présentation sommaire des caractéristiques de la région et de sa vallée, un rappel historique des travaux réalisés dans le Haut-Grésivaudan illustrera tout d'abord leurs interactions avec l'environnement.

L'aménagement du lit de l'Isère dans la traversée de l'agglomération grenobloise, étudiée récemment et en cours de réalisation, sera ensuite présentée de façon plus détaillée.

Les études concernant le maintien et même l'amélioration, pour les grandes crues, de la capacité d'amortissement à l'amont de Grenoble seront également évoquées.

\section{2 - L'Isère dans le Haut-Grésivaudan}

Il s'agit d'une section de l'Isère de $40 \mathrm{~km}$ de longueur environ, comprise entre Chapareillan, à quelques kilomètres à l'amont du pont de la Gâche et Grenoble, au pont de la Citadelle.

\section{APERCU HYDROLOGIQUE}

A l'entrée dans le département de l'Isère, cette rivière a déjà un cours de $133 \mathrm{~km}$ depuis sa source près du col de I'Iseran. Elle a reçu de nombreux affluents dont le Doron de Bozel, l'Arly, l'Arc. Certains de ceux-ci ont passé dans les retenues de barrages, en particulier ceux de Tignes, de la Sassière, du lac Noir, de Roselend, de La Girotte, d'Aussois, du Mont-Cenis. Ces réservoirs n'ont pas d'influence notable sur le régime des grandes crues.

$\mathrm{Au}$ droit de Pontcharra, elle a un bassin versant de $5200 \mathrm{~km}^{2}$. Son module est alors de $150 \mathrm{~m}^{3} / \mathrm{s}$ avec des étiages de $50 \mathrm{~m}^{3} / \mathrm{s}$ et des crues qui se produisent en général à la fonte des neiges, au printemps ou en automne (régime nivo-pluvial).

A l'heure actuelle, l'Isère, après le passage sous le pont de la Gâche, ondule faiblement au milieu de la plaine jusqu'à Lancey, puis commence à serpenter en lacets qui vont s'accentuant jusqu'à l'entrée de Grenoble, l'un des méandres particulièrement fermé, celui de Bois-Français, ayant fait l'objet d'une coupure en 1969.

Son lit est entièrement calibré par des digues qui définissent une bande de $110 \mathrm{~m}$ de largeur moyenne. Sa pente générale varie de $1,3 \mathrm{~mm}$ par mètre à Pontcharra à $0,5 \mathrm{~mm}$ par mètre à l'amont immédiat de Grenoble. A l'aval de Grenoble, et jusqu'au nouveau confluent du Drac, elle se renforce, atteignant $1,5 \mathrm{~mm}$ par mètre; plus à l'aval, on retrouve une pente de 1,3 à $1,2 \mathrm{~mm}$ par mètre. Comme 


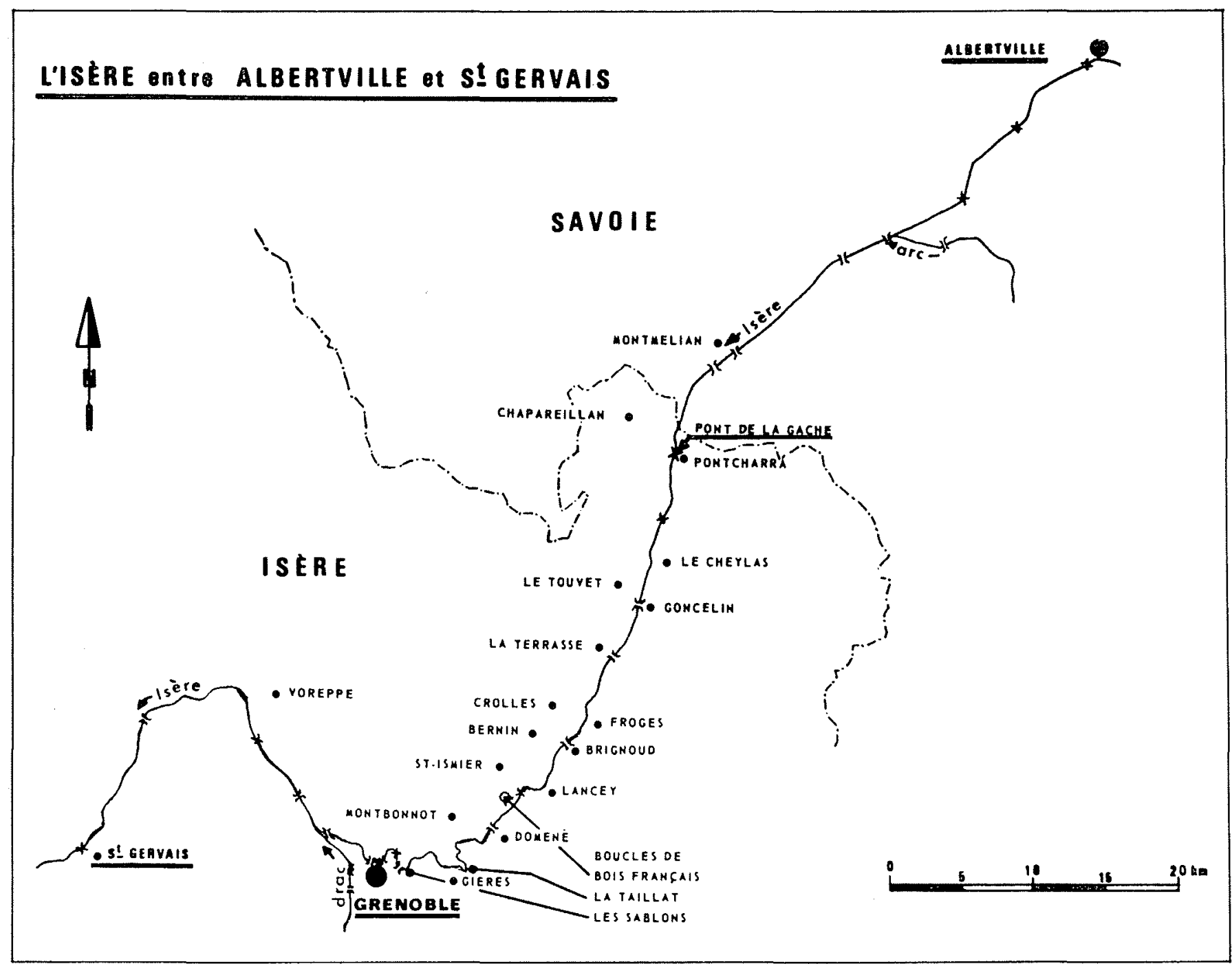

nous le verrons plus loin, cette anomalie dans le profil en long est due à la confluence du Drac. De nombreux canaux d'assainissement dénommés «chantournes» drainent les eaux de la plaine presque parallèlement au lit de la rivière, sur les deux rives, indépendamment des ruisseaux descendant des montagnes.

\section{APERCU GÉOLOGIQUE}

La vallée du Haut-Grésivaudan fait partie du profond sillon alpin qui (Gignoux et Moret) «correspond à la zone de roches tendres du Lias supérieur et des Terres noires qui, aussitôt débarrassées de leur carapace de calcaires tithoniques et crétacés, ont dû s'excaver très rapidement (sous l'action des glaciers alpins) dès le début du Quaternaire et peut-être même à la fin du Pliocène ». Il s'agit donc d'une vallée glaciaire en forme d'auge, d'une largeur de 4 à $5 \mathrm{~km}$ à son fond. Ses flancs sont constitués, rive droite, par les parois abruptes de la Chartreuse, dominés par les crêtes de calcaires urgoniens et tithoniques et dont la base jurassique est souvent recouverte de multiples éboulis et cônes de déjection. Sur la rive gauche, les pics du massif cristallin de Belledonne dominent les coteaux de schistes liasiques, auxquels sont accrochés parfois des restes de moraines glaciaires.

Le fond de vallée, de surface extrêmement plate, est formé d'alluvions quaternaires de grande profondeur - les $100 \mathrm{~m}$ sont largement dépassés à certains endroits -, constituées de graviers, sables et limons, amenés par la rivière après le retrait des glaciers. Au niveau de Grenoble, les gros galets amenés par le Drac, dont la pente du cône de déjection est de l'ordre de $3 \mathrm{~mm}$ par mètre, constituaient un seuil s'élevant plus vite que le fond de la vallée de l'Isère. C'est la présence du cône de déjection du Drac qui explique la variation de pente constatée sur le profil en long de l'Isère.

Les alluvions de l'Isère comme celles du Drac, surtout les graviers, font l'objet de certaines extractions, en vue de les transformer en matériaux de viabilité ou de matériaux à béton.

\section{APERÇU PÉdoloGiQue}

La vallée du Haut-Grésivaudan a une vocation agricole. Le climat s'y prête: froid l'hiver, chaud et humide l'été, mi-continental, mi-océanique, avec des précipitations audessus de $980 \mathrm{~mm}$ par an en moyenne (maxima en juin et 
en fin d'été-automne), bénéficiant d'une bonne protection montagneuse contre les vents d'Ouest, avec une température moyenne annuelle relativement basse et $18{ }^{\circ} \mathrm{C}$ d'écart entre le mois le plus chaud et le plus froid, ce qui donne une période de pleine activité de végétation d'avril à octobre.

Les réserves en eau du sous-sol sont en général élevées et maintiennent en profondeur une humidité souvent profitable au bon développement des cultures.

En fond de vallée, mais hors du voisinage immédiat de la rivière et de certains méandres, plus de $50 \%$ de la surface est constitué de sols peu évolués, à texture variable, à altération pédologique relativement faible, comportant des éléments calcaires et une matière organique bien humidifiée en surface. Ces sols, à porosité de 30 à $60 \%$, à perméabilité faible, ont de larges possibilités culturales, céréalières fruitières et maraîchères (en certains endroits).

Sur les terrasses basses et les cônes de déjection quaternaires, on trouve des sols bruns, calcaires, pierreux, à vocation agricole moins marquée, à faibles possibilités de cultures légumières et maraîchères, demandant moins à recevoir une irrigation d'appoint.

A certains endroits, ces terrains sont gorgés d'eau en profondeur, ce qui réduit leurs possibilités culturales à lélevage ou à certaines cultures fruitières.

Enfin, un dixième des sols sont inaptes à l'agriculture, parce que gorgés par l'eau d'une nappe aquifère à fleur de sol ou inondés fréquemment, soit par l'Isère, soit par ses torrents affluents.

\section{APERÇU SUR L'OCCUPATION URBAINE}

Sur la rive droite, les agglomérations à caractère souvent résidentiel, sont installées en chapelet, face au massif de Belledonne, le long de la RN 90, entre l'autoroute A 41 et le haut des côteaux (Chapareillan, Le Touvet, La Terrasse, Bernin, Crolles, ... Saint-Ismier, Montbonnot ...). Sur la rive gauche, on trouve un autre chapelet d'agglomérations, très différentes des précédentes, à caractère industriel fortement marqué, disposées le long du CD 523 entre la voie ferrée Grenoble-Chambéry et le débouché des vallées descendant de Belledonne, où sont nées les usines utilisant la force motrice de la houille blanche: Domène, Lancey, Froges, Goncelin, Le Cheylas, Pontcharra.

Au total, une population d'environ 35000 habitants.

\section{3 - Historique rapide des travaux d'endiguement jusqu'en 1914}

Avant le $\mathrm{XIX}^{\mathrm{e}}$ siècle, l'Isère divaguait sans contrainte dans la plaine, en larges méandres non stabilisés, changeant de lit à chaque grande crue, laissant de multiples zones marécageuses, occupant ainsi des largeurs de vallée allant parfois jusqu'à $1500 \mathrm{~m}$.

La plaine se remblayait lentement et uniformément, on pourrait même dire géologiquement, suivant les lois des phénomènes concomitants de l'érosion à l'amont du bassin versant et de la sédimentation à l'aval, des matériaux arrachés et transportés par les eaux.

Ei ceci, malgré certains efforts malheureusement trop fragmentaires des riverains qui, de tout temps, avec l'aide des ingénieurs, ont cherché à engager la lutte avec le cours d'eau pour fixer les terres cultivables, protéger les habitations, réduire les fléaux des fièvres (malaria), améliorer les communications.

Le problème n'était pas uniquement celui du département de l'Isère et, dès 1830, l'Etat Sarde avait entrepris l'endiguement complet de l'Isère et de l'Arc, avec récupération des zones marécageuses par remblaiement progressif de casiers d'atterrissement.

Faisant suite à de lointains projets, dont certains datent du XVIII $^{e}$ siècle et même au-delà, de nouveaux projets généraux d'aménagement de la plaine furent dressés, dénotant des tendances tantôt "libérale 》 dont on voit l'exemple en amont de Grenoble, tantôt « dirigiste » comme à l'aval de Grenoble.

Ces grands projets furent réalisés entre les deux guerres de 1870 et 1914 , progressivement, par un grand nombre de Syndicats de riverains, les uns nouvellement constitués, les autres datant de l'Ancien Régime, avec l'aide de subventions.

A l'amont de Grenoble, on calibra le lit, sans le modifier profondément, en laissant subsister les méandres, même à l'entrée de Grenoble (Bois-Français, La Taillat, Les Sablons), en partie à cause de la peur des habitants de cette ville de voir l'endiguement, conjugué avec une rectification (donc une augmentation de pente du lit) provoquer une aggravation des crues dans l'agglomération. Les digues furent construites sur les deux rives, protégées par des enrochements.

A l'aval de Grenoble, on redressa plus hardiment le lit à l'aide de digues parfois protégées par des perrés maçonnés.

Tout ce travail de canalisation fut complété par la création d'un vaste réseau d'assainissement de la plaine comprenant des «chantournes », des canaux, des fossés - évacuant les eaux excédentaires, imprégnant les sols à l'abri des digues - le tout entretenu par des taxes levées par les Syndicats.

Ainsi, les vallées Haut et Bas-Grésivaudan connurent un essor agricole dû à la fertilité des sols convenablement drainés et protégés.

\section{4 - Les projets et les travaux d'endiguement de 1914 à 1970}

\section{1 - DÉRoulement des traVAuX}

Cette ère de grande prospérité agricole devait avoir une fin. L'Isère, ne pouvant plus répandre sur la plaine les matériaux qu'elle transportait, les déposait uniquement dans son étroit lit mineur enfermé entre ses digues. Ces dépôts se produisaient suivant les lois de la sédimentation, en fonction de la répartition des courants de basses, moyennes ou hautes eaux et de leur vitesse d'écoulement. Des îlots de graviers et de galets abandonnés par une crue se consolidaient avec les limons qui se déposaient ensuite et se couvraient d'arbrisseaux. Ces îlots formaient barrage et arrêtaient les débits solides de la rivière, d'où un exhaussement progressif du fond du lit, plus ou moins régularisé par les crues de moyenne importance.

Cet exhaussement n'était pas régulier. Presque insensible entre Pontcharra et Goncelin comme entre Gières et le confluent du Drac, il était, au droit des méandres de Bois-Français et de La Taillat, suivant les endroits, de 
$0,80 \mathrm{~m}$ à $1,60 \mathrm{~m}$ par rapport au niveau de 1860 , ce qui correspondrait à des exhaussements moyens annuels de $0,01 \mathrm{~m}$ à $0,02 \mathrm{~m}$.

Cet exhaussement de l'Isère, pour ainsi dire «suspendu » au-dessus de la plaine, provoquait une réduction de la capacité d'écoulement du lit, qui devenait incapable d'assurer le passage des grandes crues (maximum de débit sans débordement: $700 \mathrm{~m}^{3} / \mathrm{s}$ à Bois-Français). Les digues de plus en plus souvent submergées, voire crevées, laissaient passer les flots de débits liquide et solide, ces derniers recouvrant les terres cultivées, comblant les canaux d'assainissement, qui ne remplissaient plus leur rôle de colature, et obstruant les débouchés des ouvrages. Ainsi, sous l'action d'infiltrations produites dans la plaine par la mise en charge de l'eau derrière les digues, sous celle du colmatage des "chantournes», le niveau des nappes phréatiques s'éleva, engorgeant les terres et leur faisant perdre leur valeur agricole, les transformant lentement en marécages.

L'endiguement réalisé, dont-l'effet avait été tout à fait bénéfique dans les premières années, présentait donc des inconvénients liés à la non uniformité de la capacité de charriage du chenal endigué.

En 1926 , sur $200 \mathrm{~km}^{2}$ de plaine, 50 , soit le quart, étaient manifestement atteints par excès d'humidité.

A la suite d'un concours lancé pour définir les dispositions à prendre pour enrayer le phénomène, un projet dit «Schneider » fut choisi, qui comportait, en ce qui concerne l'Isère, outre un exhaussement des digues et une remise en état des canaux d'assainissement, la coupure de trois boucles de l'Isère à l'amont de Grenoble, la création de champs d'inondation pour l'écrètement des crues, ainsi qu'un approfondissement du lit par dràgage.

Ce projet de 1929 , d'un montant de 220 millions de francs de l'époque, dont le financement était prévu à la charge de l'Etat, ne fut pas réalisé. Après le regroupement, en 1936, des syndicats de riverains en une «Association départementale », comprenant en outre la Ville de Grenoble et le département de l'Isère, une nouvelle loi autorisa l'engagement de 450 millions de l'époque de dépenses, dont $5,5 \%$ de participation des Collectivités locales, le reste à la charge de l'Etat. Rien ne fut entrepris par suite de la guerre et le projet Schneider ne fut repris qu'en 1947, après des essais sur modèle réduit pour l'étude des coupures de méandres et des recherches sur les transports solides et l'autodragage exécutés au Laboratoire Dauphinois d'Hydraulique, ancêtre de SOGREAH.

Ces essais avaient fait l'objet d'une communication présentée le 19 novembre 1946 par M. Jonte, Ingénieur en Chef des Ponts et Chaussées.

Après la crue de 1948, on établit un nouveau projet dont la réalisation s'est récemment achevée.

\section{Ce projet comportait :}

- le rétablissement en cinq ans de la capacité d'écoulement du lit de l'Isère de 1860 , en effectuant de massifs terrassements à sec par dragage dans le lit de la rivière ainsi approfondi et en utilisant les matériaux dragués pour améliorer, surélever et renforcer les digues existantes, afin d'assurer l'écoulement d'une crue de $2000 \mathrm{~m}^{3} / \mathrm{s}$ à l'amont de Grenoble et $3000 \mathrm{~m}^{3} / \mathrm{s}$ à l'aval (cube de terrassements à réaliser : $3000000 \mathrm{~m}^{3}$ environ);

- la remise en état du réseau des canaux d'assainissement de la plaine, avec aménagement des canaux existants (curage, reprofilage...), report à l'aval du débouché à l'Isère de certains canaux, pour éviter les refoulements en cas de crue, création ou reprise de plages de décantation pour les petits cours d'eau se déversant dans les canaux, enfin création de nouveaux canaux d'assainissement des zones mal drainées;

- la coupure de la boucle de Bois-Français, avec abandon du méandre de $2650 \mathrm{~m}$ de longueur et creusement d'un nouveau lit rectiligne de $850 \mathrm{~m}$, soit un raccourcissement de $1800 \mathrm{~m}$.

Les travaux de terrassements à sec ont été commencés dès 1950, mais le programme de cinq ans, d'un montant de 2 milliards de francs 1949, s'étira en longueur au rythme annuel de 100 à 150 millions d'anciens francs. Le repérage des niveaux du lit a été fait, d'abord par définition d'un équilibre de terrassements, déblai de rivière, remblai de digue, par profil tous les $80 \mathrm{~m}$, mais ensuite il fut établi par un repérage par rapport à une ligne d'eau de débit donné, notion plus hydraulique.

Une aide substantielle était apportée en même temps par des extractions commerciales, autorisées par le Service, en un certain nombre de zones, Brignoud, Montbonnot, etc.

En 1965, il avait été extrait environ $1500000 \mathrm{~m}^{3}$ au titre des terrassements à sec, réalisés pour l'Association départementale pour l'Aménagement de l'Isère et $2500000 \mathrm{~m}^{3}$ au titre des autorisations d'extraction. L'effet de ces dragages était déjà sensible puisque la crue de 1957, qui causa des dégâts si considérables en Haute-Maurienne, passa dans le Grésivaudan sans aucun débordement.

La remise en état des canaux d'assainissement était aussi une vaste opération, étant précisé que la longueur des canaux et fossés existants dans le périmètre de responsabilité de l'Association départementale de l'Aménagement de l'Isère est de $630 \mathrm{~km}$, dont 327 de canaux et fossés principaux.

Elle présentait un programme de travaux subventionnés par l'Etat (Ministère de l'Agriculture) de 8 millions de francs actuels, représentant une dépense de $410 \mathrm{~F}$ actuels pour chacun des 20000 ha de surface intéressée, dont 14000 ha de surface agricole utile $(5000$ de céréales, 4000 de prairies, 2000 ha de maïs, grains, etc.). Ce programme vient de s'achever.

Quant à la coupure du méandre de Bois-Français, elle se réalisa en 1967-1968, demandée par les populations en amont qui y voyaient un moyen d'évacuer à l'aval, par suite de la mise en vitesse provoquée par la réduction de longueur du lit, les dépôts qui s'étaient accumulés à l'amont, acceptée par la Ville de Grenoble par suite de la réduction de l'importance de ces dépôts consécutive aux extractions de matériaux faites au titre des terrassements à sec ou à titre privé. Le nouveau lit avait $0,50 \mathrm{~m}$ par kilomètre de pente, $110 \mathrm{~m}$ de large entre digues aux crêtes calées à $0,50 \mathrm{~m}$ au-dessus de la crue centenaire. Cette coupure a déjà fait l'objet d'une communication présentée par MM. Chamboredon et Belli-Riz.

\section{2 - SITUATION EN 1968 (Jeux Olympiques d'hiver)}

Ainsi, au moment de la mise en service de la coupure du méandre de Bois-Français en 1968, on pouvait penser que la lutte des habitants du Grésivaudàn allait pouvoir être ralentie, puisqu'aussi bien l'Isère et le Drac étant chacun 
maintenu entre les digues qui avaient été construites, la plaine pouvait être considérée comme étant soustraite aux effets dévastateurs des crues de ces cours d'eau, dont, par ailleurs, la période des grandes catastrophes du milieu du $\mathrm{XIX}^{\circ}$ siècle paraissait s'estomper dans un passé lointain, atténuant les craintes si vives autrefois.

La plaine avait repris un aspect d'activité agricole florissant, les marais avaient pratiquement disparu. La protection des terres agricoles, suffisamment humidifiées par une nappe phréatique maintenue à quelques mètres de profondeur, était assurée.

L'Isère et le Drac avaient leurs lits respectifs en cours d'abaissement par suite de la conjugaison, d'une part, des extractions de matériaux, les unes faisant partie des travaux de l'Association départementale, les autres autorisées pour permettre la fabrication des bétons et des matériaux routiers nécessaires à l'extension de l'agglomération grenobloise, d'autre part, de la réduction des transports solides de ces cours d'eau consécutive à la construction des grands barrages nécessaires à la production d'énergie hydro-électrique.

Cette situation aurait été satisfaisante si les abaissements de lit étaient restés de faible amplitude. Malheureusement, il n'en fut pas ainsi et ces abaissements atteignirent des chiffres de l'ordre de $0,30 \mathrm{~m}$ en moyenne par an, indépendamment des souilles profondes faites au droit des extractions commerciales.

Ces abaissements n'étaient d'ailleurs pas uniformes, dépendant essentiellement de la nature du fond du lit et de l'action de l'érosion régressive à l'amont des fonds de souilles. Ils atteignirent en certains endroits à l'amont de Grenoble, sur l'Isère, des chiffres de $2,50 \mathrm{~m}$ à $3 \mathrm{~m}$, un affaissement général du fond du lit se réalisant entre le seuil dur de Grenoble (pont de la Citadelle) et approximativement le pont de Tencin.

On distinguait nettement sur les rives de l'Isère, à l'amont de Domène, les enrochements des pieds des digues suspendues au-dessus du fond du lit, à une hauteur d'homme, parfois plus.

La situation n'aurait présenté qu'un aspect de gravité pour certains ouvrages si un phénomène, bien connu pourtant, n'était apparu en lumière: la fixité, depuis des siècles, du lit de l'Isère dans la traversée de Grenoble entre le pont de la Citadelle et le pont de la Porte-de-France, sur l'ancien confluent du Drac (avant le XIII ${ }^{\circ}$ siècle), seuil résistant, véritable pavage constitué par les galets du Drac, enrobés des limons de l'Isère.

Ainsi, aucun approfondissement ne se produisait dans cette traversée, dont les ouvrages de protection contre les crues n'étaient établis à certains endroits que pour contenir une crue de $1100 \mathrm{~m}^{3} / \mathrm{s}$. L'approfondissement important du lit en amont de la ville, sur près de $20 \mathrm{~km}$, supprimait toute possibilité d'atténuation des volumes de crue dans Grenoble, en empêchant tout débordement, voulu ou non, par-dessus ou à travers des digues (rompues) et toute constitution de zone de submersion et de rétention.

Au total, le point faible du dispositif de protection des vallées de Grésivaudan contre les crues se trouvait être la ville de Grenoble elle-même, avec toute sa population de 160000 habitants.

De nouveau, des travaux exécutés pour assainir, dans le Grésivaudan, les zones basses de la plaine, présentaient ailleurs des inconvénients et allaient nécessiter de nouvelles interventions au niveau de la traversée de l'agglomération grenobloise.

\section{5 - Aménagement du lit de l'Isère dans la traversée de Grenoble}

\section{1 - HISTORIQUE ET PRÉÉTUDES}

En 1859, la ville, ceinturée par ses fortifications, avait été entièrement noyée sous des flots dont un courant de $1500 \mathrm{~m}^{3} / \mathrm{s}$ dans le lit actuel, un autre de $300 \mathrm{~m}^{3} / \mathrm{s}$ contournant la ville par l'est et les quartiers du centre.

Depuis cette date, différents projets de défense de Grenoble furent dressés, en 1875, en 1901; les ouvrages de base en étant les digues du Drac à l'ouest, les quais de l'Isère au nord, les fortifications (aux portes obturées en cas de crue) ailleurs, avec l'idée d'un collecteur d'eau venant du centre-ville et débouchant à l'aval. Ce dernier ne fut jamais construit.

Cependant, la ville de Grenoble, éclatant dans ses fortifications, décidait de s'étendre en démolissant progressivement ces ouvrages de défense, ce qui commença en 1925 et est à peu près achevé. Les plans de défense de Grenoble étaient caducs. Il fallait d'ailleurs reprendre la question au niveau de l'agglomération grenobloise tout entière, compte tenu des constructions nouvelles: bâtiments divers, auto-' routes urbaines, nouveau pont, etc.

Un nouveau plan fut mis à l'étude. Indépendamment des informations de toutes natures fournies par les comptes rendus et les chroniques sur les crues antérieures, il était nécessaire de prendre, pour base de départ, une étude hydraulique précise, ce qui fut fait à l'aide du modèle réduit, établi en 1969 par la Société SOGREAH.

Ce modèle concernait la partie du lit de l'Isère comprise entre le pont de l'autoroute A 41 au Sablon en amont, et le confluent du Drac en aval, soit $8 \mathrm{~km}$ environ. Il s'agissait d'une réduction de la topographie des lieux au $1 / 100^{\circ}$ à échelle non distordue, en similitude de Froude, avec un fond mobile en charbon concassé, le tout équipé de vingtdeux pointes de mesure. Le modèle fut réglé à l'aide des informations sur les crues connues, en particulier à la station de jaugeage du pont Marius-Gontard, dont la courbe de tarage n'a pas changé depuis 1840 .

Les études ont ensuite consisté à faire passer sur le modèle des crues en similitude de 1200,1500 et $1800 \mathrm{~m}^{3} / \mathrm{s}$. On en déduisit des modifications à apporter aux ouvrages longitudinaux (talus, perrés, murs) et transversaux (piles et culées de ponts) ainsi qu'au lit lui-même du cours 'd'eau, afin que les écoulements d'eau précités soient convenablement assurés. On testa les modifications envisagées, en corrigeant la forme du modèle avec l'indication de ces modifications.

\section{2 - LE PLAN DE PROTECTION DE L'AGGLOMÉRATION GRENOBLOISE DE 1971}

L'objectif à atteindre est donc la protection de l'agglomération grenobloise contre la crue de l'Isère de $1800 \mathrm{~m}^{3} / \mathrm{s}$, considérée comme la crue centenaire (équivalente à celle de 1859).

Or, les études précitées ont montré que les ouvrages transversaux existants, pont de la Citadelle, passerelle suspendue, pont Marius-Gontard et pont de la Porte-deFrance, ne pouvaient laisser passer une crue de débit supéricure à $1500 \mathrm{~m}^{3} / \mathrm{s}$ sans que leurs appuis soient mis en péril et que des remous importants ne viennent provo- 
quer la submersion des défenses à l'amont. Par ailleurs, un certain nombre d'ouvrages longitudinaux, comme les quais rive gauche construits après la crue de 1859 entre l'île Verte et l'aval du pont de la Porte-de-France, étaient arasés à une cote contenant une crue de 1400 à $1500 \mathrm{~m}^{3} / \mathrm{s}$.

Ainsi, on ne pouvait, sans entraîner des dépenses très importantes hors de proportion avec le but à atteindre, concevoir de modifier ces ouvrages en superstructure. Le niveau atteint par une crue de $1500 \mathrm{~m}^{3} / \mathrm{s}$ était donc un élément de base du projet, d'autant qu'il constituait un niveau intermédiaire entre celui des premières submersions actuelles au débit de $1100 \mathrm{~m}^{3} / \mathrm{s}$ et celui de la grande crue de $1800 \mathrm{~m}^{3} / \mathrm{s}$.

Le projet comporte donc:

- un arrêt complet des extractions privées de matériaux à l'amont de Grenoble jusqu'au pont de la Gâche;

- dans la traversée de Grenoble, depuis le pont du Sablon (RN 90) jusqu'au confuent du Drac, le rehaussement des points bas des digues, perrés et murs et parapets existants, en profitant de l'occasion pour faire une remise en état quand cela est nécessaire, de telle sorte que leur arasement supérieur soit légèrement au-dessus de la ligne d'eau de la crue de $1500 \mathrm{~m}^{3} / \mathrm{s}$;

- sur toute cette longueur, avec un prolongement vers l'amont, la création d'un chenal central de $200 \mathrm{~m}^{2}$ de section $(50 \mathrm{~m} \times 4 \mathrm{~m})$ creusé par dragage dans le lit de l'Isère et permettant d'écouler un débit supplémentaire de $300 \mathrm{~m}^{3} / \mathrm{s}$, ce chenal étant muni d'un certain nombre de dispositifs;

- d'une part, des protections longitudinales en vue de stabiliser la forme de ce chenal qui aura tendance à accentuer ses courbes par les effets centrifuges des eaux, ces protections consistant en enrochements, palplanches au pied des murs, ceintures autour des piles et culées des ponts;

- d'autre part, un barrage sous-fiuvial installé en travers de l'Isère à l'aval du pont de la Porte-de-France, arasé à la cote $206000 \mathrm{NGF}$, destiné à maintenir le niveau de l'étiage à une altitude suffisante pour que la nappe phréatique, commandée par ce niveau, ne s'affaisse pas en-dessous des têtes des pieux en bois servant de fondation à de nombreux édifices de la ville.

Le montant des travaux à réaliser s'élève approximativement à 50 millions de francs.

Ce plan de défense est prévu pour être réalisé en plusieurs années, en principe en deux à trois plans quinquennaux.

Une première phase consiste à réaliser la protection contre la crue de $1500 \mathrm{~m}^{3} / \mathrm{s}$. C'est celle qui, à quelques détails près, est inscrite au VI ${ }^{\mathbf{e}}$ Plan.

Une deuxième phase ultérieure consistera à réaliser le chenal et ses protections.

Les travaux concernant la première phase sont déjà commencés. Il va sans dire que les extractions de matériaux à l'amont sont d'ores et déjà arrêtées, les entreprises ayant trouvé, en accord avec les Services, d'autres lieux d'extraction en-dehors du lit mineur. Il reste encore des extractions à l'aval de Grenoble, mais elles sont destinées à disparaître prochainement, ainsi que sur le Drac.

Il faut signaler qu'une étude complète de rentabilité économique des travaux prévus ci-dessus a été menée (SOGREAH-BCEOM), lors de laquelle ont été évalués, d'une part le coût des travaux à réaliser pour les trois types de crue précités (1 200,1500,1 $800 \mathrm{~m}^{3} / \mathrm{s}$ ), d'autre part les dégâts qui seraient occasionnés à la ville, à la population, aux industriels, aux commerçants, etc., par ces différentes crues. Cette étude a nécessité une vaste enquête auprès du public. Elle a montré que la rentabilité des travaux de protection contre la crue de $1800 \mathrm{~m}^{3} / \mathrm{s}$ était assurée largement.

En ce qui concerne le financement, l'Etat (Direction des Voies Navigables) prend une très grande part à l'opération. Pour les travaux de la première phase située à l'extérieur du centre-ville, c'est-à-dire à l'amont de la place Emée-deMarcieu et à l'aval du pont de la Porte-de-France, l'Etat, maître d'œuvre, participera à $94,5 \%$ du montant des dépenses, le reste étant réparti entre l'Association départementale, le Département de l'Isère et la Ville de Grenoble. Pour le centre-ville, où l'Association sera maîtresse d'œuvre, sa participation sera beaucoup plus faible.

Ce plan de défense comporte uniquement des travaux de protection contre les crues de l'Isère, puisque la protection contre les crues du Drac est assurée, d'autant que sa rive droite va être longée par l'autoroute B 48 presque du confluent avec l'Isère jusqu'au Pont-de-Claix, qui ajoutera et entretiendra encore ses propres ouvrages de défense.

Par ailleurs, des études ont été engagées pour mieux estimer, compte tenu des amortissements possibles à l'amont, les débits de crue à craindre à Grenoble.

\section{6 - Etude de la propagation des crues à l'amont de Grenoble}

Il a été dit plus haut que les abaissements des fonds du lit de l'Isère, particulièrement à l'amont de la coupure de Bois-Français, avaient sensiblement renforcé la valeur des débits de début de débordement à partir de laquelle l'effet d'amortissement des pointes de crue se fait sentir. D'autre part, la réalisation des axes de communication, tant ferroviaires que routiers, ont soustrait du champ dinondation des surfaces importantes. Enfin de nombreuses municipalités désirent créer, dans les zones inondables, des implantations industrielles mises hors d'eau par remblaiement, ce qui réduit d'autant les volumes accumulables sur le champ d'inondations.

En vue de cerner quantitativement l'influence que peut avoir sur les débits de pointe à Grenoble telle ou telle modification du champ d'inondation amont, la réalisation d'un modèle mathématique de la vallée de l'Isère entre Albertville et Grenoble a été confiée à SOGREAH.

$\mathrm{Ce}$ modèle est composé de casiers représentant des tronçons du lit mineur ou des portions du champ d'inondation. Les lois d'échange entre casiers contigus peuvent représenter, soit des écoulements où la perte de charge par frottement est prépondérante, soit des déversements sur des seuils noyés ou dénoyés.

A chaque pas de temps les niveaux dans chaque casier sont calculés par différences finies sur ordinateur (les termes d'inertie ne sont pas pris en compte).

Le modèle comporte, pour une longueur de vallée de $80 \mathrm{~km}$, soixante casiers pour le lit mineur, trente-six casiers en rive gauche et quarante casiers en rive droite.

En l'absence de grande crue franchement débordante depuis l'achèvement des endiguements, seul le lit mineur a pu faire l'objet d'un réglage. Une étude très soignée de la topographie des champs d'inondation, des digues longitudinales et transversales a été nécessaire pour déterminer les lois d'échange. 
Une première phase d'exploitation du modèle mathématique va permettre d'estimer l'effet d'amortissement de la plaine en présence de toute une série de systèmes d'apports introduits au confluent des principaux affiuents.

Tenant compte du fait que, dans un proche avenir, la capacité de transit à Grenoble sera portée à $1500 \mathrm{~m}^{3} / \mathrm{s}$, on examinera si les volumes d'accumulation à l'amont de Grenoble sont utilisés dans de bonnes conditions ou si des adaptations sont à apporter aux endiguements ou aux lois hauteur débit du lit mineur.

Par ailleurs, le modèle sera prêt pour tester l'incidence des multiples projets à l'étude dans les champs d'inondations, tels que projets autoroutiers, zones industrielles, etc., sur la propagation des crues.

Il permet ainsi de déterminer quantitativement les nuisances qu'un projet peut, par renforcement des débits de pointe des crues, entraîner pour les riverains situés à l'aval et éventuellement le coût des protections nécessaires qui seraient normalement à supporter par le projet.

\section{7 - Conclusion}

L'analyse des réalisations passées, plus ou moins réussies, et tout un arsenal de méthodes d'étude, permettent au Projeteur, de nos jours, de concevoir techniquement des aménagements respectant et même améliorant l'environnement. C'est en fait le coût des projets et leur financement qui posent, en général, les plus graves problèmes.

\section{Bibliographie}

«Endiguement de l'Isère et assainissement de la vallée du Grésivaudan ", Avant-projet. Mémoire de M. Cunrr, Grenoble, Imprimerie Allier (1850).

«Etudes relatives au diguement et aux inondations des rivières», par l'Ingénieur Dausse (1860-1868).

Archives dites «Fond Cunit» aux Archives départementales de l'Isère.

«L'Aménagement de la vallée du Grésivaudan». Rapport Schneider.

«Etude sur modèle réduit de la coupure des boucles de Bois-Français et de La Taillat ». Rapport 1252 du Laboratoire Dauphinoi d'Hydraulique.

«Note sur l'étude des coupures des boucles de l'Isère en amont de Grenob'e ». Communication à la SHF du 19-11-46 par M. JoNTE.

«Les travaux d'aménagement de l'Isère et de la coupure de Bois-Français sur cette rivière en amont de Grenoble ». Communication à la SHF par M. Chamboredon et M. Belli$\mathrm{RIz}$

"Etude sur modèle réduit des crues de l'Isère dans la traversée de Grenoble ». Rapport SOGREAH 10523 et 10583.

"Etude de rentabilité économique des travaux de protection contre les crues dans l'agglomération grenob'oise». Rapport SOGREAH 10729.

«Etude hydrogéologique de la plaine da Grésivaudan entre Bernin et Saint-Ismier». Rapport Cabinet Ruby.

«Etude de la nappe alluviale à l'amont de Grenoble ». Rappolt Cabinet Ruby.

«Etudes des prob'èmes hydrauliques posés par l'implantation de U. 5 entre Gières et Meylan». Rapport SOGREAH 11370.

\section{Discussion}

Président : M. J. CHAPON

M. Le Président ouvre la discussion en ces termes:

Je remercie $M$. Blanic de son exposé, qui montre combien est difficile le problème de l'aménagement d'un secteur aussi varié que celui de cette partie de l'Isère, dont la partie amont est relativement agricole et rurale et qui, plus en aval, traverse la ville de Grenoble qui a connu non pas une expansion mais une explosion.

J'ouvre la discussion: des questions sont-elles posées?

Dans quelle mesure, demande $M$. J. DE BEAuREGard (EDF), les retenues des barrages - construits depuis une cinquantaine d'années dans les bassins de l'Isère et du Drac - diminuent-elles les crues de l'lsère dans la région de Grenoble?

C'est un problème qui nous a préoccupés, répond $M$. Blanic. Pour l'Isère, les réservoirs n'intéressent que les petits bassins versants de l'amont et leur influence sur les crues est très faible au droit de Grenoble. Il n'en est pas de même pour le Drac, dont les réservoir's sont assez proches du confluent, mais, pour des raisons différentes, ils ne peuvent intervenir efficacement pour la protection de cette même zone contre les grandes crues.

Un large échange de vues auquel participent $M$. JAMME, M. VERDET, M. Morin, M. Réméniéras, M. Roche s'instaure sur l'explication de cet état de chose. I1 en ressort que :

$1^{\circ}$ Dans le cas de l'Isère, les réservoirs se trouvent trop à l'amont et intéressent une trop faible fraction du bassin versant pour être efficaces.

$2^{\circ}$ Les barrages-réservoirs sur le Drac sont exploités, en priorité, au profit d'usines hydroélectriques. S'ils peuvent amortir souvent les petites crues, et parfois les crues moyennes, ils sont impuissants en cas de crues catastrophiques. Celles-ci sont souvent dues à des épisodes pluvieux de longue durée. La première phase de la crue suffit alors à assurer le remplissage du réservoir aui se trouve plein lors du maximum de la crue.

$3^{\circ}$ D'ailleurs, le débit des organes de vidange des grands barragesréservoirs - augmenté de celui de l'usine hydroélectrique qu'ils alimentent -- est trop faible pour assurer une baisse de niveau suffisamment rapide dans le réservoir, on ve de lui permettre d'emmagasiner, en temps utile, les volumes d'eau apportés par telle ou telle phase d'une crue prévue à courte échéance.

$4^{\circ}$ Sauf dans le cas où dès la construction - comme à SerrePonçon, à Vouglans, etc. - une tranche du réservoir est réservée en permanence pour l'amortissement des crues, on ne peut compter sur les barrages hydroélectriques pour la protection contre les inondations. L'intervention efficace des différents réservoirs hydroélectriques implique la prévision des pluies et des dêbits à une échéance suffisante; malgré quelques succès spectaculaires et les efforts en cours dans ce sens, cette prévision reste encore rare et fort aléatoire.

Des études, faites par la Circonscription Electrique de Limoges, montront que les nombreux barrages-réservoirs existants dans to bassin de la Dordogne ont réduit souvent, très sensiblement, les crues faibles et moyennes mais sont restés sans grande influence sur le débit des crues exceptionnelles (celles dont la durée de retour dépasse cent ans).

M. LEFoulon souligne que, sur l'Isère, l'influence des réservoirs saisonniers, construits pour la production de l'électricité, dépend de la saison. Elle est importante sur les crues de printemps - provo. quées par de fortes précipitations et la fonte des neiges - car, à cette époque, les réservoirs sont alors systématiquement maintenus à leur plus bas niveau. Elle est pratiquement négligeable pour les 
crues d'automne, époque où le degré de remplissage des réservoirs est plus aléatoire.

Je crois pouvoir dire, conclut M. BLANIC, qu'en ce qui concerne l'Isère dans le secteur de Montmélian-Chambéry-Grenoble, nous comptons davantage, pour l'amortissement de la crue, sur l'étalement des eaux sur les grands champs d'inondation que comporte cette portion de la vallée que sur la manœuvre des réservoirs situés à l'amont. C'est ce qui fait l'intérêt du modèle mathématique dont j’ai parlé.

M. le Président remercie M. Blanic pour son exposé qui a soulevé un problème qui, dit-il, domine tout notre débat d'aujourd'hui Il tire ensuite, comme suit, les conclusions de cette demi-journée:

Le fait que nous soyons plus nombreux sur terre et de plus en plus concentrés en quelques points du territoire - notamment dans les villes, et Grenoble en est un bon exemple - donne aux problèmes de lenvironnement une dimension nouvelle, qui n'a peut-être pas été suffisamment prévue, mais avec laquelle il faut aujourdhu compter. Cela est nettement ressorti de plusieurs exposés que nous avons entendu ce matin.

La Société Hydrotechnique de France a connu ce matin une séance paticulière puisqu'on y a fait moins de théorie quo d'habitude: à vrai dire, seul le premier exposé comportait des formules et des graphiques, mais cela n'est un reproche pour personne.

Je crois que nous avons beaucoup appris ce matin - au moins, en ce qui me concerne, je reconnais avoir beaucoup appris - et cèa grâce à chacun des quatre exposés, bien différents les uns des autres par leur sujet et la façon de les aborder, et par le fait que nous avons quitté quelquefois 1'Hydraulique pure pour aborder dautres sciences plus directement humaines, et nous avons pu constater qu'avec des méthodes différentes, des problèmes ont été abordés pour faire micux et faire plus pour le bonheur des hommes.

Faire plus, cela nous est imposé directement par le fait que nous soyons plus nombreux sur terre, mais cela nous impose aussi de faire mieux. Je me suis d'ailleurs souvent posé la question : pourquoi constatons-nous, tout d'un coup, cette véritable explosion des préoccupations en matière d'environnement et de protection de la nature? Le monde a-t-il connu dans son histoire - même à des époques où les hommes ont construit de grandes choses - des réactions aussi vives contre toute création humaine et l'obligation d'un réel effort pour insérer ces créations dans la nature? Je pense que cela a dú se produire, peut-être sous d'autres formes mais probablement avec moins de force, car les hommes étaient moins nombreux et la surface de la terre étant ce qu'elle est, chacun avai une plus grande part d'espace, une plus grande part de nature. Malgré sa densité relativement faible, mais à cause de l'irrégularité dans la répartition des populations sur le territoire, la France connaît aujourd'hui des concentrations humaines qui conduisent certains habitants à considérer qu'jls vivent sur un espace restreint; le problème de l'un y concerne davantage l'autre, ce qui incite chacun à préserve plus durement son environnement individuel... au risque d'oublier que cet environnement individuel est aussi celui des autres. D'où une plus grande appréciation de la valeur de la nature où il est possible de s'évader et finalement de retrouver le modèle originel.

Ce souci ressort notamment de l'exposé de M. Clément, avec cette volonté de copier si fidèlement la nature de sorte qu'il ne soit plus possible de distinguer l'artificiel du naturel. Peut-être l'homme veut-il ainsi atteindre la perfection des perfections, en réalisant lui-même ce qui a été fait par Celui qui l'a créé.

Mais nous sommes plus nombreux et nous n'avons pas le choix, nous sommes condamnés à l'expansion économique pour tirer de la terre davantage de ressources afin de faire vivre davantage d'êtres humains - et cola avec le redoutable problème d'essayer de faira mieux vivre! - C'est ce qui ressort des quatre exposés de ce matin: le qualitatif rejoint le quantitatif dans la protection des habitants de la vallée de l'Isère, dans la création d'une voie navigable à grand gabarit dans la vallée de l'Aisne, et même sur cette petite rivière du Haut-Rhin. Nous avons vu quil existe diverses manières de protéger la nature contre les agressions humaines, mais également contre elle-mème - c'était le thème de l'excellent exposé sur la protection contre les affouillements dans les coudes des cours d'cau.

Ma conclusion sera sur une note optimiste puisque les quatre exposés, que nous avons entendus, montrent qu'il est possibje de concilier les impératifs de la croissance - et cette croissance n'a pas besoin de se faire à un rythme fou - avec la préservation de la qualité de vie, cette qualité indispensable pour que, loin de préfigurer l'enfer, les quelques années que nous avons à passer sur terre préparent le bonheur des autres.

La séance est levéc à $12 \mathrm{~h} 15$.

\section{Abstract \\ Examples of river training work along the Isère}

The river Isère winds its way down the Grésivaudan valley to Grenoble and intersects a cross-valley at Voreppe just after leaving the city. A number of river training schemes have been implemented since the mid-nineteenth century (e.g. canalisation and bank protection works), which were governed by "liberal" policies along the river above the city and a more "directorial" outlook downstream, in accordance with prevailing tendencies at the various times they were put into effect.

Major bend-cutting and dyke reinforcement work has been carried out since the middle of the twentieth century, including correction of the river's longitudinal profile, some sections of which were tending to build up by sediment deposition, whilst gravel extraction from others was unduly increasing their depth.

Work has just started on implementation of a new river flood protection programme for Grenoble. Because of considerable past and predicted river bed level variations, this programme has required more detailed research and testing than earlier schemes, which has involved fresh determination of water Jevels associated with the predicted rates of river flow, up to and including for the hundred-year peak flood discharge. For the necessary tests, a 1:100 scale model of an $8 \mathrm{~km}$ stretch of the river Isère through Grenoble was designed and run in accordance with Froude similarity laws. In addition, a mathematical model of the part of the Isère from its confluence with the river Arc to the sill at Saint Gervais, i.e. about $80 \mathrm{~km}$ in length, was subsequently designed and operated.

In designing this model, several very complicated computer calculations had to be carried out.

In addition to dyke protection and provision of a channel down the middle of the river bed, a number of sub-river sills are to be installed as a means of stabilising the river and its aquifers. Preservation of present flood areas to attenuate major flood peaks above Grenoble is also being envisaged.

In view of the highly complex problems involved on both horizontal and longitudinal scales, the physical and mathematical models in use for the study are invaluable assets as regard accurate prediction of data for present and future river engineering projects. 